\begin{tabular}{|c|l|}
\hline Title & Datta Das type spin-field-effect transistor in the nonball istic regime \\
\hline Author(s) & Ohno, Munekazu; Y oh, Kanji \\
\hline Citation & $\begin{array}{l}\text { Physical Review. B, 77(4), 045323 } \\
\text { https://doi.org/10.1103/PhysRevB.77.045323 }\end{array}$ \\
\hline Issue Date & 2008-01-22 \\
\hline Doc URL & http://hdl.handle.net/2115/32885 \\
\hline Rights & (c) 2008 A merican Physical Society \\
\hline Type & article \\
\hline File Information & GetPDFServlet.pdf \\
\hline
\end{tabular}

Instructions for use 


\title{
Datta-Das-type spin-field-effect transistor in the nonballistic regime
}

\author{
Munekazu Ohno ${ }^{1}$ and Kanji Yoh ${ }^{1,2}$ \\ ${ }^{1}$ Research Center for Integrated Quantum Electronics, Hokkaido University, Sapporo 060-8628, Japan \\ ${ }^{2}$ CREST-JST, Kawaguchi, Saitama 332-0022, Japan \\ (Received 25 June 2007; revised manuscript received 28 September 2007; published 22 January 2008)
}

\begin{abstract}
We analyzed the applicability of original Datta-Das proposal for spin-field-effect transistor (spin-FET) to nonballistic regime based on semiempirical Monte Carlo simulation for spin transport. It is demonstrated that the spin helix state in two-dimensional electron gas system is sufficiently robust against D'yakonov-Perel' spin relaxation to allow an operation of Datta-Das-type spin-FET in the nonballistic transport regime. It is also shown that the spin diffusion length of the spin helix state can be increased with an in-plane electrical field along the $[1 \overline{1} 0]$ direction. In marked contrast to early proposals for nonballistic spin-FETs, the "on" and "off" states are characterized by a $180^{\circ}$ phase difference in the spin precession motions, which is highly advantageous in terms of device flexibility.
\end{abstract}

DOI: 10.1103/PhysRevB.77.045323

PACS number(s): 85.75.Hh, 72.25.Dc, 72.25.Rb

\section{INTRODUCTION}

Understanding and controlling of carrier spin transport phenomena in a semiconductor are among the most important and interesting issues in the emerging field of spintronics because of their relevance to realization of spin-related devices. ${ }^{1}$ A seminal concept of spin-field-effect transistor (spin-FET) has been proposed by Datta and Das ${ }^{2}$ in the light of controllability of spin precession motion of electron through the Rashba spin-orbit coupling effect. ${ }^{3}$ In this device, "on" and "off" states are distinguished by $\pi$ phase difference in spin precession motion. Since a dependency of the Rashba effective magnetic field on electron wave vector $\mathbf{k}$ causes a decay of spin polarization coherence during multiple scattering events of electron, that is, D'yakonov-Perel' (DP) process, ${ }^{4}$ it is currently common belief that the DattaDas-type spin-FET can operate only in regime of ballistic transport or (quasi-) one-dimensional transport.

In a zinc blende structure, there exists the bulk-inversion asymmetry (BIA) which leads to the Dresselhaus spin-orbit coupling in the effective Hamiltonian. ${ }^{5}$ In Refs. 6 and 7, a different scenario of spin-FET operation, which is applicable to nonballistic regime, has been proposed by taking into account an interplay between the Rashba and Dresselhaus couplings. When the Rashba coupling strength is tuned to balance with the Dresselhaus strength, the effective magnetic field is oriented in [110] axis irrespective of $\mathbf{k}$ and the spread in precession motion of spin along [110] does not take place, thus leading to no spin relaxation during momentum scatterings and increasing device conductance. On the other hand, mismatch between the Rashba and Dresselhaus spin-orbit strengths causes loss of spin-related information due to the spin relaxation, which decreases the device conductance. Tuning and detuning of coupling strengths correspond to the on and off states, respectively. It can be stated that the essence of this device operation is to suppress the spin relaxation during only the on state. This idea lifts the limitation of ballistic transport or one-dimensional transport in the spinFET operation. However, it has been claimed in Ref. 8 that this nonballistic spin-FET is less preferable to the original Datta-Das-type device, since the transconductance of the nonballistic device will be roughly one-half of the transconductance of Datta-Das device. Consequently, such a nonballistic device has a large leakage current during the off state, which is approximately one-half of the on current. In the present study, we address the applicability of the original Datta-Das device to the nonballistic regime.

It has been shown in several works ${ }^{9-13}$ that the interplay between the Dresselhaus and Rashba effects causes an anisotropy in spin relaxation process so that relaxation times of spins oriented in [110], [1 10$]$, and [001] are different. When the Rashba and Dresselhaus couplings with only linear-in-k terms have equal strength, the relaxation of spin oriented in [110] axis is totally suppressed as mentioned above. On the other hand, the relaxation of spins along [1 $1 \overline{1} 0]$ and [001] occurs at a finite rate due to the DP mechanism, since the spin precession motion is involved. In our recent Monte Carlo (MC) study, ${ }^{14}$ however, it has been demonstrated that this is consequence manifested only in time-resolved analysis with assumption of homogenous spin distribution. A space-resolved MC analysis for steady state has showed that when the Rashba strength is tuned to balance with the Dresselhaus strength, the oscillation pattern of spin develops along $[1 \overline{1} 0]$ spatial coordinate without spin relaxation, that is, the relaxation of spins oriented in any axes is totally suppressed, supporting an existence of persistent spin helix (PSH) pattern recently discussed in Ref. 15. Furthermore, in Ref. 14, it has been demonstrated that the spatial distribution of coherent spin precession motion, which is observed when injected spin is oriented in [1 $1 \overline{1} 0]$ or [001], is relatively robust against the spin relaxation compared with the homogenous distribution of spin along [110] of the main concern in the nonballistic spin-FET in Refs. 6 and 7. This result is quite consistent with early theoretical study in Ref. 16, revealing that a helical spin structure has a longer spin relaxation time than a homogeneous spin distribution. Facing these facts, one may expect that the original Datta-Das proposal actually be applicable to nonballistic region in two-dimensional electron gas (2DEG) system. In this study, this possibility of nonballistic operation of the original Datta-Das device is analyzed by means of semiclassical MC simulation for spin 
transport. Our focus in this paper is placed on a specific device structure with (001) $\mathrm{In}_{0.81} \mathrm{Ga}_{0.19}$ As quantum well (QW).

The organization of this paper is as follows. In the next section, the semiclassical MC approach for spin transport is outlined, followed by results and discussion in Sec. III. We first show that the DP spin relaxation in the 2DEG system with only the Rashba coupling effect leads to a substantial loss of spin coherence. However, it will be shown that in the system with the Rashba and Dresselhaus coupling effects, the interplay between these effects strongly suppresses the DP spin relaxation for spin helix state. Then, the applicability of the Datta-Das device to nonballistic regime is addressed by analyzing the dependencies of DP spin relaxation of spin helix state on the electrical field and the momentum relaxation process. The conclusion is given in the final section.

\section{MONTE CARLO APPROACH OF SPIN TRANSPORT}

The spin relaxation during carrier transport takes place by several mechanisms such as the DP, ${ }^{4}$ Bir-Aronov-Pikus, ${ }^{17}$ Elliot-Yafet, ${ }^{18}$ and hyperfine ${ }^{19}$ mechanisms. At room temperature, among several mechanisms, the electron spin relaxation in (001) QW system is ascribable mainly to the DP mechanism. The semiclassical MC approach of electron transport has been extended to the analysis on the DP spin relaxation phenomena in Refs. 20-23. In a recent short paper, ${ }^{14}$ the DP spin relaxation process in (001) InAs QW system has been scrutinized based on such semiclassical MC approach, focusing on the difference between the spin relaxation processes observed in the time-resolved and spaceresolved analyses. The MC simulation in this paper is basically the same as the space-resolved scheme in Ref. 14, except for the inclusion of alloying scattering event. This computational scheme has not been explained in detail in Ref. 14 because of limitation of space and, therefore, more details are given below.

In an asymmetric QW system, the electron spin precession occurs along the effective magnetic field $\boldsymbol{\Omega}_{\text {eff }}(\mathbf{k})$, originating from the BIA and structure-inversion asymmetry (SIA). The corresponding spin-orbit coupling $H_{S O}$ can be expressed as $H_{S O}=(\hbar / 2) \boldsymbol{\sigma} \cdot \boldsymbol{\Omega}_{\text {eff }}(\mathbf{k}),{ }^{20}$ where $\boldsymbol{\sigma}$ is the vector of Pauli spin matrices. For the (001) QW system with the spatial coordinate of $x \|[100]$ and $x \|[010]$, the effective magnetic fields, $\boldsymbol{\Omega}_{\text {eff }}(\mathbf{k})=\boldsymbol{\Omega}_{B I A}(\mathbf{k})+\boldsymbol{\Omega}_{S I A}(\mathbf{k})$, are described within linear in $\mathbf{k}$ as

$$
\boldsymbol{\Omega}_{B I A}(\mathbf{k})=\frac{2 \gamma\left\langle k_{z}^{2}\right\rangle}{\hbar}\left(-k_{x}, k_{y}, 0\right),
$$

which is termed the Dresselhaus model ${ }^{5}$ and

$$
\mathbf{\Omega}_{S I A}(\mathbf{k})=\frac{2 \alpha}{\hbar}\left(k_{y},-k_{x}, 0\right),
$$

which is called the Rashba model. ${ }^{3}$ In Eq. (1), $\left\langle k_{z}^{2}\right\rangle$ is the expectation value of $z$ wave vector component with respect to the subband wave function and $\gamma$ is the constant of material dependent. In Eq. (2), the prefactor $\alpha$ corresponds to the
Rashba parameter depending on the material and also on the asymmetry of QW in the growth direction and is therefore controlled by the gate voltage in the spin-FET.

The spin state can be described using the following standard spin density matrix: $:^{21,23}$

$$
\rho_{\sigma}=\left[\begin{array}{cc}
\rho_{\uparrow \uparrow} & \rho_{\uparrow \downarrow} \\
\rho_{\downarrow \uparrow} & \rho_{\downarrow \downarrow}
\end{array}\right],
$$

where $\rho_{\uparrow \uparrow}\left(\rho_{\downarrow \downarrow}\right)$ represents the probability of finding electron with up-spin (down-spin) state. The off-diagonal matrix elements, $\rho_{\uparrow \downarrow}$ and $\rho_{\downarrow \uparrow}$, characterize the degree of superposition of up-spin and down-spin states. During free flight motion of electron, the spin density matrix undergoes the following unitary evolution:

$$
\rho_{\sigma}(t+\Delta t)=e^{-i H_{S O}(\mathbf{k}) \delta t / \hbar} \rho_{\sigma}(t) e^{i H_{S O}(\mathbf{k}) \delta t / \hbar} .
$$

It is important to note that the spin polarization component is given as $S_{n}=\operatorname{Tr}\left[\sigma_{n} \rho_{\sigma}\right]$ with $n=x, y$, and $z$ and the unitary evolution given in Eq. (4) can be recast in the following equation of classical momentum $\mathbf{S}$ under the effective magnetic field as explained in Refs. 20 and 21,

$$
\frac{\partial \mathbf{S}}{\partial t}=\boldsymbol{\Omega}_{e f f}(\mathbf{k}) \times \mathbf{S} .
$$

The reciprocal effect of spin on the electron motion through spin-orbit coupling is neglected in the present calculations, as is common in the reported works. ${ }^{20-23}$ In the present study, the orientation of spin polarization vector is described in [110], [1 10$]$, and [001] coordinate systems and the corresponding vector components are represented by $S_{+}, S_{-}$, and $S_{z}$, respectively.

During the free flight motion of an electron, the evolution of spin polarization vector is described by Eq. (5) in which the effective magnetic field is $\mathbf{k}$ dependent. Within the semiclassical MC approach, the electron motion following classical trajectories is interrupted by the scattering events, yielding new direction and magnitude of electron momentum, and repeating this process on an electron ensemble provides the evolution of wave vector during multiple scattering events, which therefore enables the direct observation of the DP spin relaxation process. ${ }^{14,20-23}$

In this study, we focus on the DP process at $300 \mathrm{~K}$ for a (001) $\mathrm{In}_{0.81} \mathrm{Ga}_{0.19}$ As QW of $80 \AA$ well width inserted between $\mathrm{In}_{0.53} \mathrm{Ga}_{0.47} \mathrm{As}$ subchannel and $\mathrm{Al}_{0.48} \mathrm{In}_{0.52} \mathrm{As}$ barrier layer, which is one of the most promising structures for realizing spin-FET as detailed in Ref. 24. The channel composition of $\mathrm{In}_{0.81} \mathrm{Ga}_{0.19}$ As is determined based on the limitation of possible metamorphic growth on InP substrate. Figure 1(a) represents the spatial profiles of conduction band and square of wave function at zero gate voltage, $V_{g}=0$. The Rashba parameter $\alpha$ in InAs QW system is known to take a relatively large value ${ }^{25}$ and it is therefore expected that in the present structure with channel layer of InAs-rich composition, large values of $\alpha$ are associated with a moderate range of gate voltage $V_{g}$. The structure is subjected to Si $\delta$ doping with the concentration of $2.0 \times 10^{12} \mathrm{~cm}^{-2}$ at $50 \AA$ above the $\mathrm{Al}_{0.48} \mathrm{In}_{0.52} \mathrm{As} / \mathrm{In}_{0.81} \mathrm{Ga}_{0.19} \mathrm{As}$ heterointerface, and the carrier concentration in the channel is calculated to be 9.1 
(a)

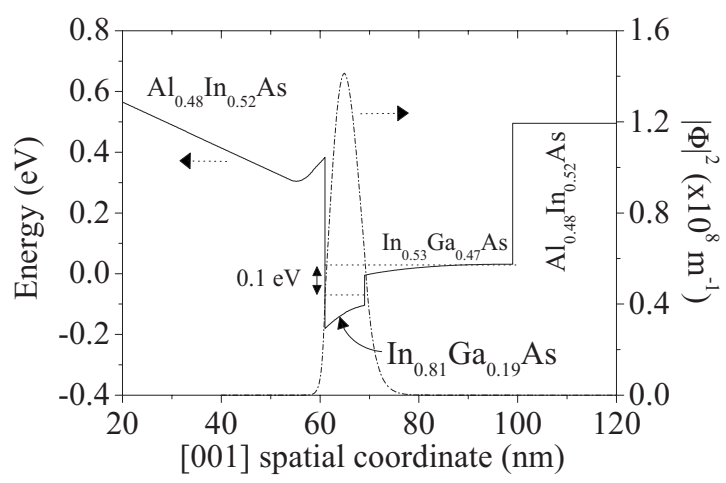

(b)

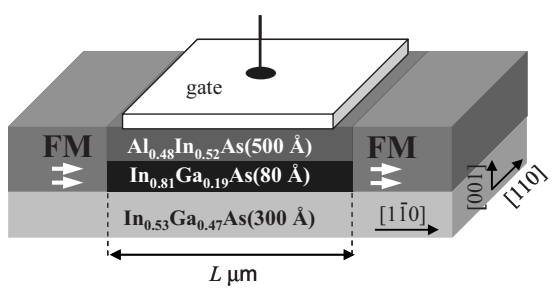

FIG. 1. (a) Calculated spatial profiles of conduction band (left vertical axis) and square of wave function (right vertical axis) at $V_{g}=0$. (b) Schematic picture of the present spin-FET structure.

$\times 10^{11} \mathrm{~cm}^{-2}$. The difference between first and second subband energies is $0.1 \mathrm{eV}$. In the present MC simulation, for simplicity, we employed one-subband approximation.

The device structure of our focus is schematically represented in Fig. 1(b). The gate length $L$ is in micron and the gate width is assumed be to infinite. The ferromagnetic electrodes, whose polarizations are assumed to be $100 \%$, are magnetized in the [1 $\overline{1} 0]$ direction. It is stressed that when the Rashba and Dresselhaus couplings have equal strength, i.e., $\alpha=\gamma\left\langle k_{z}^{2}\right\rangle$, the effective magnetic field is oriented in [110] axis, irrespective of $\mathbf{k}$. Hence, if the injected spin is parallel to [110] axis, the spin experiences neither spin precession nor relaxation. ${ }^{6}$ On the other hand, in the present device, the injected spin is parallel to [1]̄0] axis and hence the spin precession is involved in electron traveling through the channel layer. In the present calculation, the spatial distribution of electron over [110] and [001] coordinates is assumed to be uniform, and our concern is placed on one-dimensional observation along [1피 spatial coordinate between the drain and source. As will be discussed later, this current direction is essential for the spin-FET operation to utilize the spin helix state in (001) QW system. When the electron reaches the drain, it is removed from the channel and is injected at the source. After a steady state in the electron momentum distribution is reached, the spin polarization along the [1 $\overline{10}]$ axis is assigned to the injected electron at the source, i.e., $S_{-}=1$ at $r_{110}=0$. The spin polarization is averaged over some period at each [1 $\overline{1} 0]$ spatial coordinate and this averaged value is denoted as $\langle\mathbf{S}\rangle_{T}$.
The present ensemble MC simulation uses $4 \times 10^{4}$ electrons. The momentum scatterings included in this simulation are elastic scatterings due to polar optical phonon, acoustic phonon, remote impurity, and alloy scattering, for which the scattering rates in the $2 \mathrm{DEG}$ system are explicitly given in Ref. 26. The carrier mobility at $V_{g}=0.0$ is calculated to be $9.70 \times 10^{3} \mathrm{~cm}^{2} /(\mathrm{V} \mathrm{s})$ in the present $\mathrm{MC}$ simulation, which is quite comparable to the $9.75 \times 10^{3} \mathrm{~cm}^{2} /(\mathrm{V} \mathrm{s})$ value experimentally found for the same structure. ${ }^{24}$ It was found that in the present structure, the variation of $V_{g}$ does not yield significant changes in all the scattering rates expect for the alloy scattering rate. Negative gate voltage decreases the electron density in the 2DEG system and accordingly the screening constant, leading to an increase in the alloy scattering rate. The carrier mobility decreases with gate voltage. For instance, the carrier mobility at $V_{g}=-0.5 \mathrm{~V}$ is calculated to be $8.84 \times 10^{3} \mathrm{~cm}^{2} /(\mathrm{V} \mathrm{s})$.

As shown in Eqs. (1) and (2), the strengths of Dresselhaus and Rashba effects are entirely determined by the constants $\gamma$ and $\alpha$, respectively. In all the calculations, the Dresselhaus parameter $\gamma$ is assumed to be $59 \times 10^{-30} \mathrm{eV} \mathrm{m}^{3}$ which is compositionally averaged value of $71 \times 10^{-30} \mathrm{eV} \mathrm{m}^{3}$ for InAs (Ref. 27) and $10 \times 10^{-30} \mathrm{eV} \mathrm{m}^{3}$ for GaAs. ${ }^{27}$ The constant $\beta$ given as $\beta=\gamma\left\langle k_{z}^{2}\right\rangle$ is calculated to be 9.2 $\times 10^{-12} \mathrm{eV} \mathrm{m}$ for the present QW system. As for the Rashba parameter $\alpha$, we vary this value systematically using a ratio of $\alpha / \beta$ which we call the Rashba-Dresselhaus (RD) ratio hereafter. Our focus is placed on the range of RD ratio between 0 and 3 which corresponds to the variation of $\alpha$ between 0 and $27.6 \times 10^{-12} \mathrm{eV} \mathrm{m}$. This is reasonable range of $\alpha$ value in the InAs-rich channel layer. ${ }^{25,28}$ It should be mentioned that the Rashba parameter $\alpha$ is practically controlled by the gate voltage $V_{g}$ and hence there exists a one-to-one correspondence between the values of $\alpha$ and the momentum scattering rate which also depends on $V_{g}$. However, our calculations demonstrated that the difference in the scattering rate does not cause a substantial change in the spatial profile of spin polarization within the range of $\mathrm{RD}$ ratio considered here, as will be discussed later. This fact allows one to analyze the dependence of spin relaxation process on the RD ratio using the scattering rates independently calculated at a fixed value of gate voltage. For the sake of simplicity, the scattering rates at $V_{g}=0.0$ are employed for all the calculations, except for the one shown in Fig. 4.

\section{RESULTS AND DISCUSSION}

Figure 2(a) represents the spatial profiles of spin polarization component $\langle S\rangle_{T}$ plotted with respect to the source-drain distance and the RD ratio. The gate length is set to be $2 \mu \mathrm{m}$ and the in-plane field $E_{d s}$ is $3.0 \mathrm{kV} / \mathrm{cm}$. For comparison, the spin relaxation processes calculated with the same condition but without the Dresselhaus term, which is equivalent to the original Datta-Das proposal, are represented in Fig. 2(b). The Rashba parameter in Fig. 2(b) is normalized by 9.2 $\times 10^{-12} \mathrm{eV} \mathrm{m}$ which is equal to the value of $\beta$ in Fig. 2(a). The spatial oscillation of spin polarization is observed in all the cases. As shown in Fig. 2(b), during the spin transport with only the Rashba coupling effect, the coherence of spin 
(a)

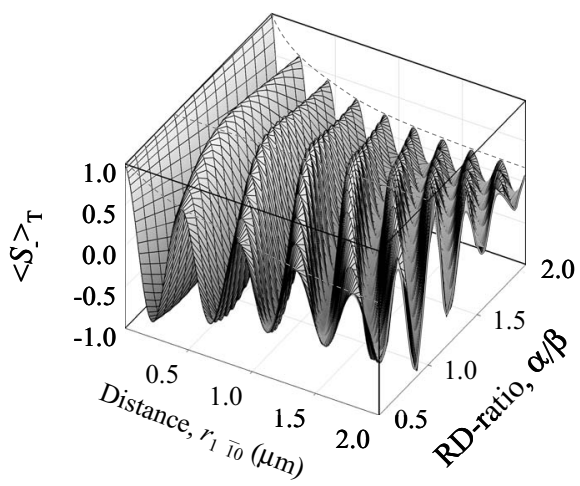

(b)

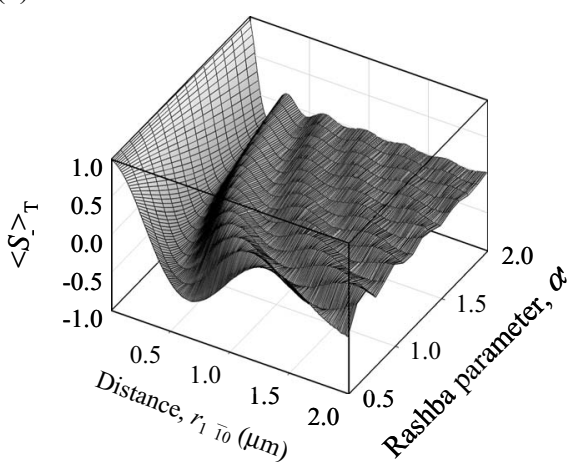

FIG. 2. (a) Spin polarization component $\left\langle S_{-}\right\rangle_{T}$, with respect to source-drain distance $r_{1 \overline{1}}$, and RD-ratio calculated at $E_{d s}$ $=3.0 \mathrm{kV} / \mathrm{cm}$. (b) Spin polarization component $\left\langle S_{-}\right\rangle_{T}$ with respect to distance $r_{110}$ calculated with the same condition as the ones in (a) but without the Dresselhaus coupling, which is equivalent to the original Datta-Das proposal.

polarization is almost completely lost at $L=2 \mu \mathrm{m}$ because of strong spin relaxation. In the system with the Rashba and Dresselhaus effects [Fig. 2(a)], on the other hand, the coherence of spin polarization at any RD ratio remarkably persists even at $L=2 \mu \mathrm{m}$. From the comparison between Figs. 2(a) and 2(b), it can be readily grasped that the interplay between the Rashba and Dresselhaus couplings strongly suppresses the spin relaxation. Importantly, no spin relaxation takes place at $\alpha / \beta=1.0$ and this spatial pattern is exactly equivalent to the PSH state of which the existence has been pointed out in Ref. 6 and recently discussed based on SU(2) symmetry of spin-orbit Hamiltonian in Ref. 15.

Within the evolution equation of spin precession motion given in Eq. (5), the precession angle of spin during a certain time period $\Delta \tau$ is expressed as

$$
\theta=\left|\Omega_{e f f}(\mathbf{k})\right| \cdot \Delta \tau=\frac{2 m^{*} r}{\hbar^{2}} \sqrt{\alpha^{2}+\beta^{2}-2 \alpha \beta \sin 2 \theta},
$$

where $m^{*}$ is the electron effective mass, $r=\hbar\left(k_{x}^{2}\right.$ $\left.+k_{y}^{2}\right)^{1 / 2} \Delta \tau / m^{*}$ is the magnitude of moving distance of electron from the source, and $\theta$ is the angle of wave vector with respect to $x$ axis. When the relation $\alpha=\beta$ is realized, the precession angle is reduced to

$$
\theta=\frac{4 \alpha m^{*}}{\hbar^{2}} r_{1 \overline{10}}
$$

where $r_{110}^{-}=r \cos \left(\theta+\frac{\pi}{4}\right)$ is the moving distance of electron along $[1 \overline{1} 0]$ spatial coordinate. The precession angle is thus proportional to the displacement of electron along the [1 $\overline{10}]$ spatial coordinate, which is the origin of the PSH states. ${ }^{29}$ The effective magnetic field parallel to [110] does not cause the spin relaxation during electron transport along the [1]0] direction. Therefore, it can be stated that the spin relaxation at $\alpha \neq \beta$ is entirely ascribable to the existence of effective magnetic field of which the orientation deviates from [110] axis. Although the Rashba effective field is always perpendicular to $\mathbf{k}$, the interplay between the Rashba and Dresselhaus effects makes the effective field parallel to [110] dominant in the vicinity of $\alpha / \beta=1.0$, thus suppressing the spin relaxation, as observed in Fig. 2(a). This fact is the key to the successful operation of the Datta-Das device in the nonballistic regime.

In Fig. 2(a), the dashed lines at $\alpha / \beta=2.0,1.0$, and 0.5 represent the magnitude of spin polarization vector given as $|\langle\mathbf{S}\rangle|_{T}=\left(\left\langle S_{+}\right\rangle_{T}^{2}+\left\langle S_{-}\right\rangle_{T}^{2}+\left\langle S_{z}\right\rangle_{T}^{2}\right)^{1 / 2}$ which is a measure of the coherence of spin polarization. The spatial profile of $|\langle\mathbf{S}\rangle|_{T}$ at any value of RD ratio is well described by an exponential decay, viz., $|\langle\mathbf{S}\rangle|_{T}=\exp \left(-r_{110} / l_{S}\right)$ with a characteristic "spin diffusion length" $l_{s}$. At $\alpha / \beta=1.0, l_{s}$ become infinite, denoting the PSH, as discussed for InAs channel in Ref. 14. The spatial profiles of $\left\langle S_{-}\right\rangle_{T}$ calculated at several in-plane fields are shown in Fig. 3(a). The RD ratio is set to be 1.5. It is seen that the increment of $E_{d s}$ leads to suppression of spin relaxation, while a period of spatial oscillation is independent of the in-plane field. The dependency of spin diffusion length on the in-plane field at several RD ratios is plotted in Fig. 3(b). The spin diffusion length increases with the in-plane field, which is quite similar to the behavior of "downstream" spin diffusion length defined in the case of homogeneous spin distribution within a drift-diffusion approach. ${ }^{30,31}$ With increasing the in-plane field, the number of electrons contributing to current transport increases at $\mathbf{k} \|[1 \overline{1} 0]$ where $\boldsymbol{\Omega}_{\text {eff }}(\mathbf{k})$ is always oriented to [110] irrespective of the $\mathrm{RD}$ ratio, thus suppressing the spin relaxation in the spin helix state.

It is well known that the DP spin relaxation time $\tau_{s}$ is given as $\tau_{s} \propto 1 / \tau_{p}$ with the momentum relaxation time $\tau_{p}$ (Ref. 4) and, hence, the increment of momentum scattering increases the spin relaxation time $\tau_{s}$, that is, the motional narrowing effect. ${ }^{1}$ Within the drift-diffusion approach, then, the spin diffusion length $l_{s}=\left(D \tau_{s}\right)^{1 / 2}$ is independent of the momentum relaxation time $\tau_{p}$ because of $D \propto \tau_{p}$. ${ }^{1}$ First, it is pointed out that although there have been a number of theoretical investigations on the anisotropic behavior of spin relaxation rate, ${ }^{9-13}$ the spin relaxation time for the spin helix state has never been appropriately characterized, as discussed in Ref. 14. The robustness of the spin helix state against the DP relaxation can be observed in the space-resolved analysis of the present focus, and the dependence of the spin diffusion length for the helix state on the momentum relaxation process is worth discussing. In our MC simulation, the momen- 
(a)

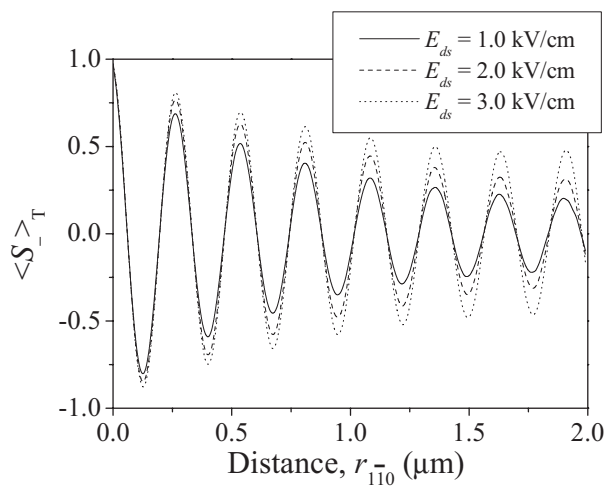

(b)

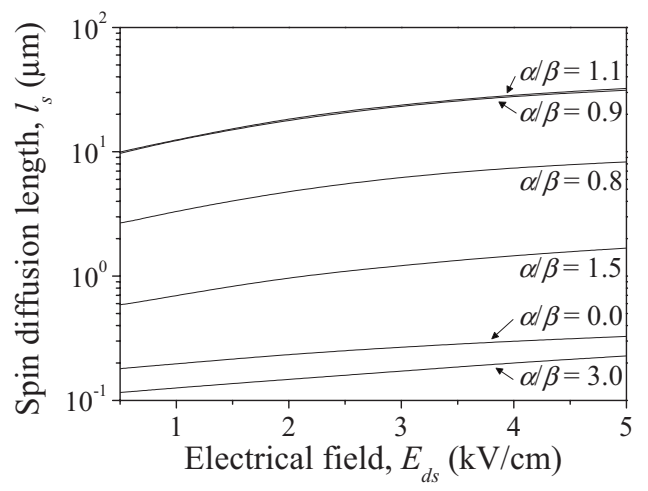

FIG. 3. (a) Spatial profile of spin polarization component $\left\langle S_{-}\right\rangle_{T}$ calculated at several $E_{d s}$. The RD ratio is given as $\alpha / \beta=1.5$. (b) Dependence of spin diffusion length $l_{s}$ on the in-plane electrical field $E_{d s}$ calculated at several RD ratio.

tum scattering event takes place due to polar optical phonon, acoustic phonon, remote impurity, and alloy scatterings. The DP spin relaxation during different momentum relaxation processes was calculated by changing these scattering rates through the variation of the gate voltage or by changing them arbitrarily. It was demonstrated that the spatial profile of spin polarization is almost invariant irrespective of the difference in the momentum relaxation processes. As shown in Fig. 4, the spin diffusion length is essentially independent of the electron mean free path $l_{p}$. The slight increment of $l_{s}$ can be

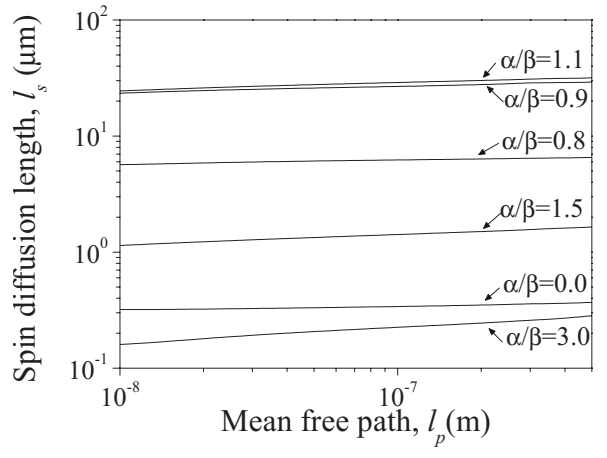

FIG. 4. Dependence of the spin diffusion length on mean free path of electron at several RD ratio. (a)

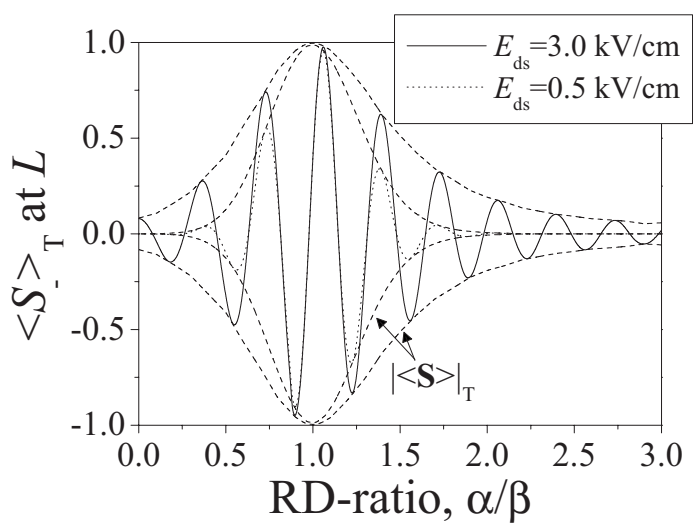

(b)

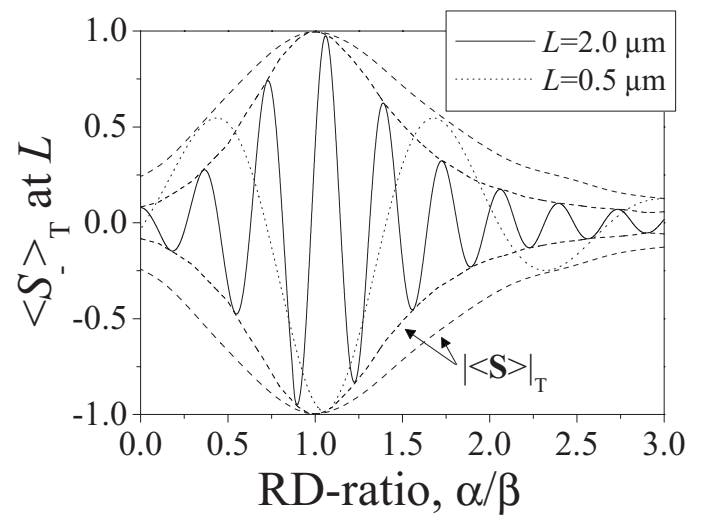

FIG. 5. (a) Spin polarization component $\left\langle S_{-}\right\rangle_{T}$ at $L=2 \mu \mathrm{m}$ calculated at different $E_{d s}$. The solid and dotted lines indicate the results at $E_{d s}=3.0$ and $0.5 \mathrm{kV} / \mathrm{cm}$, respectively. The dashed lines denote $|\langle\mathbf{S}\rangle|_{T}$ at each $E_{d s}$. (b) Spin polarization component $\left\langle S_{-}\right\rangle_{T}$ at $E_{d s}=3.0 \mathrm{kV} / \mathrm{cm}$ calculated at different $L$. The solid and dotted lines indicate the results at $L=2.0$ and $0.5 \mu \mathrm{m}$, respectively.

explained by the increment of the number of electrons contributing to current transport at $\mathbf{k} \|[1 \overline{1} 0]$ in the high mobility field. From Fig. 4, it can be mentioned that the present discussion has a generality in that the spin relaxation process shown in this paper should be quite commonly observed in a wide range of carrier mobility and a difference could appear only when the value of $\beta$ is changed.

Based on these MC results, we address the nonballistic operation of the Datta-Das device. First, as shown in Fig. 2(a), the number of peaks in spatial oscillation decreases with decreasing the $\mathrm{RD}$ ratio. It is quite important to note that an oscillation can be seen at a fixed spatial coordinate by changing the RD ratio, which is associated with the current oscillation due to variation of the gate voltage in the spinFET. Such an oscillation of $\left\langle S_{-}\right\rangle_{T}$ at $L=2 \mu \mathrm{m}$ is demonstrated by the solid line in Fig. 5(a) where the magnitude of spin polarization vector $|\langle\mathbf{S}\rangle|_{T}$ is also shown as a dashed line. In the present device, the on and off states correspond to upper and lower peaks in this oscillation, respectively. The period of oscillation $\Delta \alpha / \beta$, which does not obviously change 
over this range of $\mathrm{RD}$ ratio, is about 0.15 and this is entirely associated with the variation of the gate voltage required to switch on and off states. Importantly, the amplitude of oscillation denoted as $\Delta\left\langle S_{-}\right\rangle_{T}$ in the vicinity of $\alpha / \beta=1.0$ is close to maximum value (2.0) realized without the spin relaxation. Hence, the Datta-Das device can successfully operate in the nonballistic regime. In Fig. 5(a), moreover, the calculated result at $E_{d s}=0.5 \mathrm{kV} / \mathrm{cm}$ is represented by the dotted line. The peak positions are identical with the ones at $E_{d s}$ $=3.0 \mathrm{kV} / \mathrm{cm}$. Although the amplitude of oscillation becomes small and vanishes at low and high values of $\alpha / \beta, \Delta\left\langle S_{-}\right\rangle_{T}$ in the vicinity of $\alpha / \beta=1.0$ is considered sufficiently large to allow the successful operation of the device.

It is generally expected that $\Delta\left\langle S_{-}\right\rangle_{T}$ should be large in a device with smaller gate length, since the coherence of spin polarization persists. In Fig. 5(b), the oscillation of spin at $L=2 \mu \mathrm{m}$ is compared with the one at $L=0.5 \mu \mathrm{m}$. As expected, the magnitude $|\langle\mathbf{S}\rangle|_{T}$ at $L=0.5 \mu \mathrm{m}$ is always larger than the one at $L=2 \mu \mathrm{m}$. However, $\Delta\left\langle S_{-}\right\rangle_{T}$ becomes small at $L=0.5 \mu \mathrm{m}$. From Eq. (6), $\Delta \alpha / \beta$ required for $\pi$ rotation at $\alpha=\beta$ is obtained as $\Delta \alpha / \beta=\pi \hbar^{2} /\left(2 m^{*} \beta L\right)$. Hence, $\Delta \alpha / \beta$ increases with decreasing gate length $L$, which results in the peak positions for the on and/or off states deviated far from $\alpha / \beta=1.0$, leading to the decrement of $\Delta\left\langle S_{-}\right\rangle_{T}$, as seen in Fig. $5(\mathrm{~b})$. In other words, the long gate length results in small $\Delta \alpha / \beta$ and thus relatively large $\Delta\left\langle S_{-}\right\rangle_{T}$ because both the on and off states are located in the vicinity of $\alpha=\beta$.

The difference between the nonballistic devices proposed in Refs. 6 and 7 and in this work is emphasized. In the nonballistic device proposed in Refs. 6 and 7, the spin relaxation process is suppressed only during the on state by tuning $\alpha$ to balance with $\beta$, viz., $\alpha=\beta$. This suppression of spin relaxation can utilize either the PSH or spatially homogeneous spin distribution in the channel. ${ }^{6}$ On the other hand, the off state is realized by detuning of $\alpha$ which leads to the spin relaxation and thus a decrease in device conductance. As claimed in Ref. 8, in this device with a relatively long channel, the electron spins arriving at the drain are equally likely to be parallel or antiparallel to the magnetization vector in the ferromagnetic drain, and the minimum value of the off conductance is approximately one-half of the on conductance. More importantly, this device has a large leakage current during the off state, which is roughly considered to be one-half of the on current. Another device setup has been pointed out in Ref. 6, based on switching between two points $\beta= \pm \alpha$, which means that both the on and off states do not suffer from the spin relaxation process. However, this switching operation between $\beta= \pm \alpha$ generally requires a large variation of the gate voltage. The present MC simulation demonstrates that the strong suppression of the DP process in the spin helix state is realized not only at $\alpha / \beta=1.0$ but also over a wide range of $\alpha$ value, i.e., even at $\alpha \neq \beta$, and the $\pi$ phase difference of spin precession motion can sufficiently be utilized to distinguish the on and off states in the nonballistic regime. Especially, if the PSH at $\alpha / \beta=1.0$ is utilized for the off state, the off current becomes zero in an ideal case and, moreover, the on current is not substantially reduced due to the spin relaxation. This can be seen, for example, by tuning the gate length. Figure 6 shows the os-

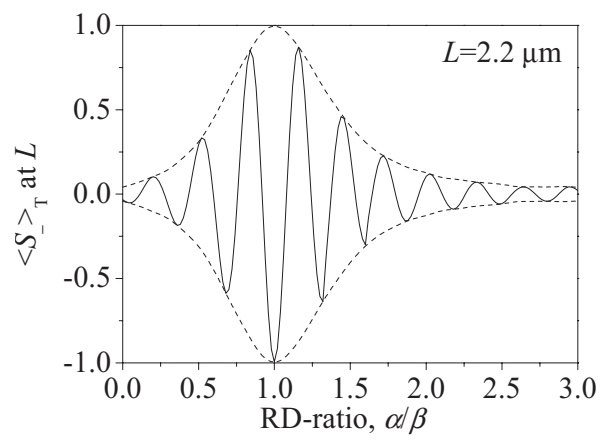

FIG. 6. Spin polarization component $\left\langle S_{-}\right\rangle_{T}$ (solid line) and magnitude of spin polarization $|\langle\mathbf{S}\rangle|_{T}$ (dashed line) at $L=2.2 \mu \mathrm{m}$ calculated at different $E_{d}=3.0 \mathrm{kV} / \mathrm{cm}$.

cillation of $\left\langle S_{-}\right\rangle_{T}$ at the drain calculated with $L=2.2 \mu \mathrm{m}$. One can see that $\left\langle S_{-}\right\rangle_{T}$ becomes 0 at $\alpha / \beta=1.0$ which corresponds to the off state and, on the other hand, $\left\langle S_{-}\right\rangle_{T}$ at $\alpha / \beta=1.17$ or 0.83 reaches about 0.85 which is associated with the on current. It is therefore considered that the application of the Datta-Das spin-FET to the nonballistic regime be preferable to the early proposal of nonballistic spin-FET.

In the present calculations, we ignored the cubic term in Dresselhaus model. The cubic term causes a decay of the PSH state and the spin diffusion length becomes finite even at $\alpha=\beta .{ }^{15}$ Therefore, the off current is not completely zero in the present device. The cubic term effect in the present device operation should be seriously addressed and also the device structure needs to be optimized for reducing such a cubic term effect. Furthermore, details of injection and detection of spin polarized current were not taken into account in the present work. In this regard, we would like to refer to experimental works ${ }^{32,33}$ where successful injection of spin polarized current into InAs channel has been demonstrated. Finally, it should be mentioned that the value of RD ratio focused in the present study has been experimentally observed in several types of structures such as InAs/InAlAs and GaAs/AlGaAs. ${ }^{34}$

\section{CONCLUSION}

In this study, the applicability of Datta-Das-type spin-FET in the nonballistic regime was analyzed based on MC simulation of spin transport. It was demonstrated that the DP spin relaxation in the 2DEG system with only the Rashba coupling effect leads to a substantial loss of spin coherence and, in the system with the Rashba and Dresselhaus coupling effects, however, the interplay between these effects strongly suppresses the DP spin relaxation for spin helix state. More importantly, the strong suppression of the DP spin relaxation for spin helix state is realized not only at $\alpha / \beta=1.0$ but also over a wide range of $\alpha$ value, i.e., even at $\alpha \neq \beta$. Furthermore, the spin diffusion length of the spin helix state can be

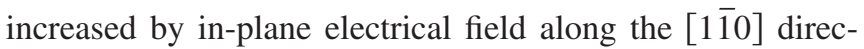
tion, which can be explained by an increase in the number of electrons contributing to current transport at $\mathbf{k} \|[1 \overline{1} 0]$. Then, 
the $\pi$ phase difference of spin precession motion can be used to distinguish between the on and off states in the nonballistic regime of electron transport along the [1피 direction. This fact suggests that the Datta-Das device can successfully operate in the nonballistic regime of spin transport in a 2DEG system, contrary to common belief. This Datta-Dastype nonballistic spin-FET is characterized by better figures of merit (on and/or off conductance ratio) compared to early proposals for nonballistic spin-FETs.

\section{ACKNOWLEDGMENT}

This work was partly supported by Grant-in-Aid for Scientific Research from the Japanese Ministry of Education, Culture, Sports, Science and Technology.
${ }^{1}$ I. Zutic, J. Fabian, and S. D. Sarma, Rev. Mod. Phys. 76, 323 (2004).

${ }^{2}$ S. Datta and B. Das, Appl. Phys. Lett. 56, 665 (1990).

${ }^{3}$ Y. A. Bychkov and E. I. Rashba, JETP Lett. 39, 78 (1984).

${ }^{4}$ M. I. D'yakonov and V. I. Perel', Sov. Phys. Solid State 13, 3023 (1972).

${ }^{5}$ G. Dresselhaus, Phys. Rev. 100, 580 (1955).

${ }^{6}$ J. Schliemann, J. C. Egues, and D. Loss, Phys. Rev. Lett. 90, 146801 (2003).

${ }^{7}$ X. Cartoixà, D. Z.-Y. Ting, and Y.-C. Chang, Appl. Phys. Lett. 83, 1462 (2003).

${ }^{8}$ S. Bandyopadhyay and M. Cahay, Appl. Phys. Lett. 85, 1433 (2004)

${ }^{9}$ N. S. Averkiev and L. E. Golub, Phys. Rev. B 60, 15582 (1999).

${ }^{10}$ N. S. Averkiev, L. E. Golub, and M. Willander, J. Phys.: Condens. Matter 14, R271 (2002).

${ }^{11}$ J. Kainz, U. Rössler, and R. Winkler, Phys. Rev. B 68, 075322 (2003).

${ }^{12}$ Y. V. Pershin, Physica E (Amsterdam) 23, 226 (2004).

${ }^{13}$ J. Cheng and M. W. Wu, J. Appl. Phys. 99, 083704 (2006).

${ }^{14}$ M. Ohno and K. Yoh, Phys. Rev. B 75, 241308(R) (2007).

${ }^{15}$ B. A. Bernevig, J. Orenstein, and S. C. Zhang, Phys. Rev. Lett. 97, 236601 (2006).

${ }^{16}$ Y. V. Pershin, Phys. Rev. B 71, 155317 (2005).

${ }^{17}$ G. L. Bir, A. G. Aronov, and G. E. Pikus, Sov. Phys. JETP 42, 705 (1976).

${ }^{18}$ R. J. Elliot, Phys. Rev. 96, 266 (1954); Y. Yafet, in Solid State Physics, edited by F. Seitz and D. Turnbull (Academic, New York, 1963), Vol. 14.

${ }^{19}$ A. W. Overhauser, Phys. Rev. 92, 411 (1953).

${ }^{20}$ A. A. Kiselev and K. W. Kim, Phys. Rev. B 61, 13115 (2000).
${ }^{21}$ S. Pramanik, S. Bandyopadhyay, and M. Cahay, Phys. Rev. B 68, 075313 (2003).

${ }^{22}$ A. Bournel, P. Dollfus, E. Cassan, and P. Hesto, Appl. Phys. Lett. 77, 2346 (2000).

${ }^{23}$ M. Shen, S. Saikin, M. C. Cheng, and V. Privman, Math. Comput. Simul. 65, 351 (2004).

${ }^{24}$ K. Yoh, M. Ferhat, A. Riposan, and J. M. Mullunchick, in Physics of Semiconductors, Proceedings of the 27th International Conference on the Physics of Semiconductors, edited by J. Menéndez and C. G. Van de Walle (American Institute of Physics, New York, 2005).

${ }^{25}$ D. Grundler, Phys. Rev. Lett. 84, 6074 (2000).

${ }^{26}$ D. H. Park and K. F. Brennan, J. Appl. Phys. 65, 1615 (1989).

${ }^{27}$ W. Knap, C. Skierbiszewski, A. Zduniak, E. Litwin-Staszewska, D. Bertho, F. Kobbi, J. L. Robert, G. E. Pikus, F. G. Pikus, S. V. Iordanskii, V. Mosser, K. Zekentes, and Yu. B. Lyanda-Geller, Phys. Rev. B 53, 3912 (1996).

${ }^{28}$ Th. Schäpers, G. Engels, J. Lange, Th. Klocke, M. Hollfelder, and H. Lüth, J. Appl. Phys. 83, 4324 (1998).

${ }^{29}$ M. H. Liu, K. W. Chen, S. H. Chen, and C. R. Chang, Phys. Rev. B 74, 235322 (2006).

${ }^{30}$ Z. G. Yu and M. E. Flatte, Phys. Rev. B 66, 201202(R) (2002).

${ }^{31}$ Z. G. Yu and M. E. Flatte, Phys. Rev. B 66, 235302 (2002).

${ }^{32}$ K. Yoh, H. Ohno, Y. Katano, K. Sueoka, K. Mukasa, and M. E. Ramsteiner, Semicond. Sci. Technol. 19, S384 (2004).

${ }^{33}$ H. C. Koo, H. Yi, J. B. Ko, J. Chang, S.-H. Han, D. Jung, S.-G. Huh, and J. Eom, Appl. Phys. Lett. 90, 022101 (2007).

${ }^{34}$ S. Giglberger, L. E. Golub, V. V. Bel'kov, S. N. Danilov, D. Schuh, C. Gerl, F. Rohlfing, J. Stahl, W. Wegscheider, D. Weiss, W. Prettl, and S. D. Ganichev, Phys. Rev. B 75, 035327 (2007). 